\title{
Facilitators and barriers to detection and treatment of depression, anxiety and experiences of domestic violence in pregnant women attending Midwife Obstetric Units (MOUs) in Cape Town, South Africa: A qualitative study
}

Zulfa Abrahams ( $\nabla$ zulfa.abrahams@uct.ac.za )

University of cape Town https://orcid.org/0000-0002-7336-6009

Sonet Boisits

Marguerite Schneider

Simone Honikman

Crick Lund

Research Article

Keywords: barriers; facilitators; depression; pregnant; health system; healthcare

Posted Date: May 10th, 2021

DOl: https://doi.org/10.21203/rs.3.rs-512424/v1

License: (a) This work is licensed under a Creative Commons Attribution 4.0 International License.

Read Full License 


\section{Abstract \\ Background}

In South Africa, symptoms of common mental disorders (CMDs) such as depression and anxiety are highly prevalent during the perinatal period and linked to experiences of domestic violence. However, limited routine detection and treatment is available to pregnant women with these problems. We investigated facilitators and barriers of service-providers and -users in detecting and treating pregnant women with symptoms of CMDs and experiences of domestic violence.

\section{Methods}

Service-provider perspectives were informed by qualitative interviews with 37 healthcare workers providing care to pregnant women attending four midwife obstetric units (MOUs) in Cape Town. Qualitative interviews with 38 pregnant women attending the same MOUs for their first antenatal care visit provided service-user perspectives.

\section{Results}

Facilitators identified included the availability of a mental health screening questionnaire and the perceived importance of detection and treatment. Barriers included service providers' heavy workload and discomfort with discussing mental health issues; lack of standardised referral pathways and poor uptake of referrals; lack of confidentiality and feelings of shame related to experiences of domestic violence.

\section{Limitations:}

Difficulty in linking perceptions of care with specific healthcare providers; social desirability bias pregnant women's responses to questions on domestic violence, and service providers' responses to their role in providing care.

\section{Conclusion}

The facilitators and barriers identified indicate the need to strengthen health systems by training antenatal care nurses to detect symptoms of CMDs and experiences of domestic violence in pregnant women, developing standardised referral pathways and training lay healthcare workers to provide treatment for mild symptoms of depression and anxiety.

\section{Introduction}


Common mental disorders (CMDs), such as depression and anxiety, are highly prevalent during the perinatal period, with low- and middle-income countries (LMIC) carrying the greatest burden. It is estimated that in LMIC, $18 \%$ of perinatal women experience depression (Woody, et al, 2017) and 34\% experience anxiety (Dennis, et al, 2017). In South Africa, the prevalence of depressive symptoms during pregnancy ranges between 27\% and 39\% (Abrahams, et al, 2018; Hartley, et al, 2011; Manikkam and Burns, 2012; Redinger, et al, 2018), while symptoms of anxiety range between $15 \%$ and $23 \%$ (Redinger, et al, 2018; van Heyningen, et al, 2017). Antenatal depression is associated with adverse infant and child outcomes, such as preterm birth, low birth weight, infant stunting and underweight children (Gelaye, et al, 2016; Jarde, et al, 2016; Rahman, et al, 2007). In LMIC, evidence suggests that screening and treating CMDs during pregnancy improves the health and economic outcomes of mothers and their children (Baranov, et al, 2020; Rahman, et al, 2013), and has been suggested as a key approach to improving the health of perinatal women and children in South Africa (Tomlinson, et al, 2014).

As a result of a growing awareness of the burden of disease and economic costs associated with mental disorders, South Africa adopted the Mental Health Policy Framework (MHPF) and Strategic Plan 20132020 (Department of Health, 2013) which aligns with the WHO Mental Health Action plan (World Health Organisation, 2012). However, having a new policy framework supporting the integration of mental health into primary healthcare (Docrat, et al, 2019) has not led to the desired changes in service delivery, and the detection of symptoms of CMDs continue to be excluded from routine care provided at public healthcare facilities in South Africa (English, et al, 2017).

Little is known about the facilitators and barriers to detection, referral and treatment of pregnant women with depression, anxiety and experiences of domestic violence in public sector healthcare facilities in South Africa. The Health System Strengthening in sub-Saharan Africa (ASSET) study (ASSET, 2020) has undertaken to work with the Western Cape Department of Health (DoH) to develop and evaluate the impact of a screening, referral and counselling intervention for pregnant women with CMDs and experiences of domestic violence. As part of the development of the intervention, this study investigated the facilitators and barriers to detecting and treating pregnant women with symptoms of CMDs and experiences of domestic violence attending public sector healthcare facilities in Cape Town.

\section{Methods}

\section{Setting}

In South African primary healthcare (PHC) facilities, antenatal care is provided by nursing staff as well as a number of lay healthcare workers, such as HIV counsellors and breastfeeding counsellors. The DoH also funds non-profit organisations (NPOs), mandated to provide facility- and community-based support to patients attending PHC facilities. NPOs employ teams of lay community healthcare workers (CHWs) who are managed by a professional nurse, called an outreach team leader (OTL). 
This qualitative study was conducted in four midwife obstetric units (MOUs) in Cape Town, South Africa, and in the NPOs providing community-based support in the area surrounding the MOUs. MOUs were purposively selected by sub-district $\mathrm{DoH}$ managers to represent the 4 sub-districts in the Cape Metro health district. All MOUs are managed by the Western Cape DoH and offer free antenatal, birthing and postnatal services to pregnant women. The MOUs are situated on the 'Cape Flats' (South African History Online, 2020) - a large, flat area covered by sandy soil on the outskirts of the city of Cape Town. The area consists of Black townships and Coloured [people of mixed ancestry (Adhikari, 2005)] ghettos where these communities were forcibly moved to as a result of Apartheid laws in South Africa. These communities experience high levels of unemployment, poverty (Statistics South Africa, 2017), substance abuse (Pluddemann, 2012), gang violence (Maringira and Masiya, 2018) and domestic violence (Malan, et al, 2018).

\section{Data collection}

Healthcare workers providing care to pregnant women, working in four MOUs and for the NPOs providing support to them, were purposively selected and recruited between August and December 2019. Managers were asked to provide the names of key staff members involved in the care of pregnant women at each MOU and the supporting NPOs. The following cadres of healthcare workers from each of the four MOUs were invited to participate in semi-structured interviews: operational managers, antenatal care (ANC) nurses, breastfeeding counsellors, HIV counsellors, health promotion officers, mental health nurses, social workers, CHWs and OTLs. All except one health promotion officer agreed to participate in the study.

During November 2019 and June 2020, a facility-based healthcare worker provided pregnant women attending the four MOUs for their first antenatal visit with an ASSET study information sheet, available in participants' home language. They were asked to read the information sheet while waiting for their routine assessments to be completed. After at least 30 minutes, a researcher approached the pregnant women and invited them to participate in the study, and if they agreed, to complete a mental health screening. Those who consented were screened for symptoms of CMDs using the Edinburgh Postnatal Depression Scale (EPDS) (Cox, et al, 1987) and a 3-question mental health screening questionnaire (Abrahams, et al, 2019). In addition, participants were screened for experiences of domestic violence using a brief 3-question screening questionnaire. The violence screening questionnaire used a 12-month recall period and asked about experiences of verbal or emotional abuse (e.g., shouting or swearing, name calling, insulting, threats of intimidation, ignoring or excluding, isolating, humiliating, blaming), physical abuse (e.g., beating, pushing, kicking, biting, slapping) and sexual abuse (e.g., forced sexual intercourse or intercourse without your consent). The EPDS, using a 7-day recall period, has been validated in South Africa with a cut-off $\geq 13$ indicating a probable CMD (De Bruin, et al, 2004; Lawrie, et al, 1998). The 3question mental health screening questionnaire comprised of three mood questions (Abrahams, et al, 2019), derived from the Whooley scale (Whooley, 2016), the Generalised Anxiety Scale (GAD-2) (Skapinakis, et al, 2007), and the Edinburgh Postnatal Depression Scale (EPDS) (Cox, et al, 1987). The questionnaire, which uses a two-week recall period, was validated against the EPDS and found to 
correctly classify $91 \%$ of women screened using a cut-point of $\geq 2$, making it highly sensitive (sensitivity=85.7) and specific (specificity=92.9) (Abrahams, et al, 2019). Pregnant woman who scored $\geq 13$ on the EPDS, $\geq 2$ on the 3-question mental health screening questionnaire or $\geq 2$ on the domestic violence screening questionnaire were invited to participate in a semi-structured interview. Recruitment continued at all facilities until saturation was reached.

English language, semi-structured interview guides were developed, and translated to Afrikaans and IsiXhosa by bilingual experts. The interviews were administered by trained researchers in English, Afrikaans and IsiXhosa, took between 30 and 60 minutes to complete, and were audio-recorded. All interviews were conducted in a private space, with no others present, to ensure confidentiality. Healthcare workers were asked about their role and attitude to detecting, referring and treating pregnant women with symptoms of depression or anxiety, and experiences of domestic violence (Supplementary File 1). Pregnant women were asked about their understanding of depression, anxiety and experiences of domestic violence, and their perceived reasons for experiencing psychological distress (Supplementary File 2). All participants were asked about their thoughts on the acceptability and feasibility of a routine screening and counselling service for pregnant women.

\section{Data analysis}

The semi-structured interviews that were conducted in English were transcribed verbatim, while interviews conducted in Afrikaans or IsiXhosa were translated into English and transcribed by bilingual speakers. A framework analysis approach (Gale, et al, 2013) was used to analyse the transcripts. The development of initial codes was guided to a certain degree by the semi-structured interview topics. Further themes not captured by the initial coding were identified through extensive reading of the transcripts and coding passages interpreted as important. Transcripts and data were managed using NVivo 12 Pro qualitative data analysis software (QSR International Pty Ltd) (Bazeley and Jackson, 2013).

Ten percent of the healthcare workers transcripts were randomly selected for analysis by three researchers, and the inter-rater reliability assessed (agreement $=78.4 \%$; Gwet's agreement coefficient (AC) (Gwet, 2014; Klein, 2018) =0.7329). Coding disagreements were discussed and resolved. Thereafter, each of the three researchers analysed a third of the remaining transcripts.

Similarly, $10 \%$ of the pregnant women's transcripts were randomly selected for analysis by two researchers, and the inter-rater reliability assessed (agreement $=87.3 \%$; Gwet's AC=0.8547). Coding disagreements were discussed and resolved. Thereafter, each researcher analysed half the remaining transcripts.

\section{Ethical approval}


Ethical approval for the study was obtained from the Human Research Ethics Committee at the University of Cape Town (Ref No: 139/2018) and the Psychiatry, Nursing and Midwifery Research Ethics Subcommittee at King's College London (Ref No: 17/18-7807). In addition, the Western Cape Department of Health approved the use of the research sites (Ref No: WC_201807_008). Those who participated in the study provided written, informed consent after the procedure had been verbally explained to them. All participants were informed that they were free to withdraw from the study at any time without consequences. Pregnant women who screened positive for CMDs or experiences of domestic violence, were offered a referral to the on-site mental health nurse or social worker. Pregnant women who participated in the semi-structured interviews were given a food voucher valued at ZAR100. No financial incentives were provided to healthcare workers.

\section{Results}

Semi-structured interviews were conducted with 37 healthcare workers who provided care to pregnant women or managed staff who provided the care (Table 1). The majority of healthcare workers were female $(91.9 \%)$, older than 35 years $(75.7 \%)$, employed by an NPO $(56.8 \%)$ and worked in a healthcare facility (67.5\%).

Table 1: Demographic characteristics of Healthcare workers $(n=37)$ 


\begin{tabular}{|ll|}
\hline Characteristics & Healthcare workers \\
\hline Gender $(\%)$
\end{tabular}

* Individuals of mixed ancestry (Adhikari, 2005)

One hundred and fifty-six pregnant women were screened for symptoms of CMDs and experiences of domestic violence. Thirty-eight women (24\%) screened positive and were invited to participate in a semistructured interview (Table 2). The majority of women interviewed were 26 years or older $(55.2 \%)$ and were unemployed (44.7\%). Almost all the women interviewed scored $\geq 2$ on the mental health screening questionnaire (94.7\%), while $29 \%$ ( $n=11)$ scored $\geq 2$ on the domestic violence screening questionnaire. 
The facilitators and barriers identified by healthcare workers and pregnant women have been organised into three categories: system-level, provider-level and patient-level (Table 3). The facilitators and barriers will be presented under two main headings representative of the stages along the care pathway: (1) detection of CMDs and experiences of domestic violence, and (2) referral and treatment of CMDs and experiences of domestic violence.

Table 2: Demographic and clinical characteristics of pregnant women $(n=38)$

\begin{tabular}{|ll|}
\hline Characteristics & Pregnant Women $\mathrm{n}(\%)$ \\
\hline Age & \\
\hline 19-25 years & $6(15.8)$ \\
\hline $26-35$ years & $11(29.0)$ \\
\hline $36-40$ years & $17(44.7)$ \\
\hline Race & $4(10.5)$ \\
\hline Coloured* & \\
\hline Black & $26(68.4)$ \\
\hline Employment status & $12(31.6)$ \\
\hline Employed & $12(31.6)$ \\
\hline Unemployed & $17(44.7)$ \\
\hline Student & $9(23.7)$ \\
\hline Gestation & \\
\hline First trimester & $15(39.5)$ \\
\hline Second trimester & $19(50.0)$ \\
\hline Third trimester & $4(11.5)$ \\
\hline Previous pregnancies & $21(55.3)$ \\
\hline None & $5(18.4)$ \\
\hline 1-3 pregnancies & $24(63.2)$ \\
\hline$>3$ pregnancies & \\
\hline Scored $\geq 13$ on EPDS** & $10(29.3)$ \\
\hline Scored $\geq 2$ on the mental health screening questionnaire & \\
\hline Scored $\geq 2$ on domestic violence screening questionnaire & \\
\hline
\end{tabular}


*Individuals of mixed ancestry (Adhikari, 2005)

**Edinburgh Postnatal Depression Scale

Table 3: System-, provider- and patient-level facilitators and barriers to detection, referral and treatment for common mental disorders (CMDs) and experiences of domestic violence

\section{Facilitators Barriers}

\section{Detection of CMDs and experiences of domestic violence}

Availability of a mental health screening questionnaire

System-level

Aspects of the mental health screening questionnaire

System-level

Lack of a domestic violence screening questionnaire

System-level

Perceived importance of detection

Provider-

level

Patient-level

Perceived lack of confidentiality

Patient-level

Heavy workload

Providerlevel

Discomfort with mental health issues

Providerlevel

Referral and treatment of CMDs and experiences of domestic violence

Lack of availability of referral pathways

System-level

Poor awareness of referral pathways

Provider-

level

Patient-level

Perceived importance of counselling

Provider-

level

Patient-level

Cultural beliefs and stigma

Patient-level

\section{Detection of CMDs and experiences of domestic violence}

\section{Tools for detection}


Varying rates of antenatal mental health and domestic violence screening were reported across the four facilities. The Perinatal Mental Health Project (PMHP) (Honikman, et al, 2012) supported one of the facilities by providing an on-site screening and counselling service to all pregnant women attending the MOU for their first antenatal visit. As part of the service, a health promotion officer screened all pregnant women at their first antenatal visit for symptoms of CMDs and the risks thereof. Women who screened positive were referred to the on-site counsellor. The PMHP reported detecting many women with symptoms of depression and anxiety - "I guess six out of ten [screen positive for depression], ..... and three or four out of ten [screen positive for anxiety]" (Health Promotion Officer), while the other facilities detected fewer women - "I see two or three on a weekly basis" (Health Promotion Officer), and "It's now and then. It's very rare..." (ANC Nurse). During the data collection period, an updated version of the Maternity Case Record (MCR) (Department of Health, 2018) - the national stationery used to record all aspects of the pregnancy - was released. The updated MCR contained a mental health screening questionnaire (Abrahams, et al, 2019) (the same 3-question mental health screening questionnaire used in this study to detect women with symptoms of CMDs), to be administered by ANC nurses at the patients' first clinic visit.

Detecting women with experiences of domestic violence was less common in all facilities. Healthcare workers reported that they did not specifically enquire about domestic violence. Experiences of domestic violence were only detected when a physical examination revealed signs of physical abuse, or when the woman voluntarily disclosed the information. One ANC nurse reported detecting women who were abused "twice or thrice a month", while an MOU manager reported that detecting women who were abused was "not often, but we do". A health promotion officer admitted that if she specifically enquired about domestic violence, she would detect many women as "domestic violence is so high here in this area", and that "they [pregnant women] would be more vulnerable".

\section{Perceived importance of detection}

All, except for one of the facility-based healthcare workers interviewed, felt that it was important to screen pregnant women for symptoms of depression and anxiety, and experiences of domestic violence. One mental health nurse made the link between symptoms of CMDs that was left untreated and the consequences to the mother and child by saying: "I think it's very important that these things needs to be picked up because it can only get worse when the baby is born, for both of them. You know, for the baby and for the mother's...mom's sake". Many healthcare workers felt that screening for CMDs should occur at all antenatal and postnatal clinic visits. One ANC nurse mentioned that CMDs could occur at any point during the perinatal period, not just when women attended the MOU for the first time as symptoms of depression and anxiety "can start any time during pregnancy. It can even be later in pregnancy."

\section{Perceived lack of confidentiality}


While the majority of pregnant women reported that they would be happy to disclose their feelings of depression and anxiety, or experiences of domestic violence, a few women expressed their concerns regarding confidentiality. One pregnant woman was especially concerned about whether such sensitive information would remain confidential by saying: "... especially in a community where everyone knows everyone, I do not trust them [nurses]". Some healthcare workers also expressed doubts about whether the pregnant women would disclose their feelings by saying: "... remember people put up a nice face hey, they don't want people to know how they feeling" (Breastfeeding Counsellor).

In the context of the four facilities, many women were financially dependent on their partners, and were concerned about disclosing their experiences of physical abuse. A breastfeeding counsellor explained why women who were abused rarely spoke about their experiences by saying: "Some of them are ashamed that they're actually having to tell you this, where other people is not experiencing it, but they are experiencing it. Now they're ashamed". One pregnant woman referred to the possible consequences of disclosing her abuse by saying "... so we have to think twice before you do something that you are going to regret".

\section{Heavy workload}

All ANC nurses were concerned about the amount of time it took to assess patients' mental health as their heavy workload and large patient numbers allowed them limited time with each patient. One ANC nurse highlighted her heavy workload in relation to her role as a midwife by saying: "I can't [screen all patients] because now it's gonna take me a long time. Cause the thing is, I'm busy with a patient, ... then you worry, I need to see the next patient.... so I won't be giving much time to her. I will only just be very...just so, just on the top". In addition, ANC nurses reported feeling concerned that they would not be able to provide a comprehensive consultation if they had the added responsibility of asking women about their mental health. One ANC nurse chose to wait for patients to mention that they were feeling distressed, instead of administering the screening questions to all patients - "... it's a full clinic now, you already stressing, you want to finish that patient. ... if she's not gonna ask you or tell you about, tells you about her problem then you oversee that - you overlook that, because she looks happy, she's not looking stressful. Then you, then you just will leave it..." Many of the ANC nurses were also concerned about the amount of time it took to contain a patient who felt distressed as a result of administering the mental health screening questionnaire. One ANC nurse reflected on her experiences by saying: "sometimes they will start opening up and then it's a problem. You open a can of worms. Cause those questions are thought-provoking....if they're gonna start crying...they'll start crying obviously because they'll start thinking sister, that and that...And then for me it sounds rude to just okay, I'm gonna send you to ...."

\section{Discomfort with mental health issues}

Many ANC nurses felt uncomfortable discussing mental health issues with patients. They thought of themselves as clinicians who were primarily responsible for the physical well-being of the pregnant 
woman and her foetus, and that they were not trained to adequately address mental health issues. One ANC nurse described the discomfort she experienced when discussing issues related to mental health by saying: "I can talk and everything but there's a point where right, I'm not trained for this ... I don't want to take in so much because it also takes so much out from you". Other healthcare workers were concerned that they were not trained to manage women who screened positive - "if you probe and you ask those three questions [the mental health screening questionnaire], what do you do if the answer is yes?" (ANC Nurse).

\section{Referral and treatment of CMDs and experiences of domestic violence}

\section{Lack of availability of referral pathways}

Referral pathways varied across the four facilities and depended on the issue requiring referral. Patients with symptoms of depression or anxiety were referred to the mental health nurse, a social worker, a registered psychologist (if available) or to the district hospital, while those who felt suicidal were referred to the mental health nurse, a medical officer, the trauma unit or to a registered psychologist. Women who reported experiencing domestic violence were most often referred to the social worker, if one was available. However, specialists such as social workers, mental health nurses and registered psychologists carried a heavy workload and could often only see women who were referred a few weeks later. While some healthcare workers were aware of the referral pathway in their facility, many were not. One lay healthcare worker voiced her uncertainty by saying: "I do not even sometimes know where to refer the mothers to" (HIV Counsellor). The majority of pregnant women were also unaware of how to access mental health care, or whether it was available at the healthcare facilities. A pregnant woman expressed her frustration by saying: "I don't know how I can get help and where...".

\section{Perceived importance of counselling}

Both healthcare workers and pregnant women thought that providing a counselling service was important. One facility-based healthcare worker expressed her thoughts on the importance of counselling by saying: "... many women who are untreated, or undiagnosed or not caught in the system do end up later on in life here at psychiatry or even with worse symptoms and now it is not only affected themselves but now also the baby after its being born" (Mental Health Nurse). One pregnant woman explained why counselling was important by saying: "I think it would be good, yes, because talking about it makes you feel much better and for me talking to a stranger is much better than talking to people that knows you".

\section{Cultural beliefs and stigma}


While the importance of providing a counselling service was highlighted, healthcare workers also pointed out that many women who were referred for mental health counselling, declined the offer when they were told to see a mental health nurse, registered psychologist or social worker. Healthcare workers conveyed some of the reasons women had given them for declining the offer of counselling. One ANC nurse explained that mental health issues were stigmatised in the community and that patients were concerned that they would be branded as "...mad if you go for counselling". A lay healthcare worker explained that the community was very small, and that patients were concerned that "... they [community members] look at others and they point fingers" (CHW). One healthcare worker highlighted the role that cultural beliefs played, by explaining that in some Black and Coloured cultures, counselling was not acceptable. When these women were referred for counselling, they declined saying: "... [they] must just be okay" (ANC Nurse).

Healthcare workers blamed the normalisation of psychological and physical abuse in the community for the poor uptake of referrals by women who were abused, whereas pregnant women attributed their financial dependence on their partner or husband, as the reason for declining a referral. Healthcare workers thought that women who were abused believed that the abuse was "... how he is showing his love" (Breastfeeding Counsellor), or that he "did not mean to hurt them" (ANC Nurse), or that the women "deserve it" (Breastfeeding Counsellor). One pregnant woman referred to the normalisation of domestic violence in her community by saying: "... it [the abuse] is not a big thing for them [family and friends], because it happens a lot". Many pregnant women who were abused felt that a referral for mental health counselling would not help them as it would not be able to change their dependence on their abusive partners. One pregnant woman explained that she had accepted her abusive relationship by saying: "Everyone mos [anyway] has their own problems ...". Another pregnant woman explained that counselling could not change her situation as "I am not working. He is the breadwinner; I must be submissive".

\section{Discussion}

This paper utilizes qualitative semi-structured interviews with healthcare workers and pregnant women, to identify the facilitators and barriers to mental health care for pregnant women attending four MOUs in Cape Town. We found that while healthcare workers and pregnant women acknowledged the importance of detecting women with symptoms of depression and anxiety, or experiences of domestic violence, detection rates were low. Barriers contributing to the low detection rates included patients' concerns about the lack of confidentiality and feelings of shame related to experiences of domestic violence; and healthcare workers discomfort in dealing with mental health issues, their limited time available and heavy patient load. Interviews with healthcare workers highlighted the lack of standardised referral pathways and the poor uptake of referrals by women with symptoms of depression and anxiety, or experiences of domestic violence.

Several studies have reported the high prevalence of symptoms of CMDs (Abrahams, et al, 2018; Garman, et al, 2019; Schneider, et al, 2018; van Heyningen, et al, 2016) and experiences of domestic violence (Davis, et al, 2017; Malan, et al, 2018; Schneider, et al, 2018) in perinatal women attending public 
healthcare facilities in South Africa. We found that $63 \%$ of the women screened had symptoms of a CMD and $29 \%$ had experienced domestic violence. Yet our study found that few women were detected by healthcare workers and linked to care.

Several system-, provider- and patient-level facilitators and barriers were identified. The availability of a mental health screening questionnaire, included in the updated MCR (Department of Health, 2018) released during the data collection period, was identified as a system-level facilitator, as it enabled nurses to screen pregnant women for CMDs as part of their routine consultations. However, several system-level barriers linked to the MCR were also identified: the mental health screening questionnaire contains a caveat, indicating that the screening should only be conducted if "resources are available for referral, e.g. mental health nurse, social worker, NGO, medical officer, counsellor, psychiatrists or other services"; the MCR only allows for the mental health screening questionnaire to be completed at the first consultation with the patient; and no questions are available to detect experiences of domestic violence (Department of Health, 2018). As depression and anxiety may develop (Malan, et al, 2018) and remit (Garman, et al, 2019) at any point during the perinatal period, only administering the screening questionnaire at the first clinic visit, would result in missed opportunities for care for women who develop symptoms of depression or anxiety later in their pregnancy, or after they have given birth. While the MCR provides a list of counselling topics, including one for domestic violence, the lack of a screening questionnaire for detecting domestic violence could be responsible for the poor detection rates found in communities where domestic violence is prevalent (Field, et al, 2018; Malan, et al, 2018), as studies suggest that routine screening for domestic violence in primary healthcare settings improves detection (Chen, et al, 2013; Sivarajasingam, 2021). The final system-level barrier contributing to the low detection and referral rate, was the lack of standardised referral pathways. We found that both healthcare workers and pregnant women were unclear about how and where to access care. As the mental health screening questionnaire was only to be conducted when resources were available, healthcare workers who were unaware of resources available in the facility, were free to opt out of providing the screening service to perinatal women.

Provider-level facilitators included the perceived importance of detecting pregnant women with CMDs and experiences of domestic violence, and the treatment of women who screened positive. In this study, healthcare workers acknowledged the importance of providing a screening, referral and counselling service. However, several provider-level barriers were identified. ANC nurses who were tasked with conducting the screening felt that they were not the right cadre of staff to provide the service. ANC nurses cited their large patient load, the additional time needed to screen patients, their lack of awareness of the referral pathway, and their discomfort with mental health issues as the primary reasons for their reluctance. Studies investigating the challenges to providing quality healthcare in South Africa have highlighted the shortage of healthcare workers, especially at the nursing level in urban areas (Maphumulo and Bhengu, 2019). As it is unconstitutional to deny anyone access to basic healthcare in public healthcare facilities in South Africa (Coovadia, et al, 2009), these facilities are often overcrowded with inadequate staff and resources available to provide quality care to all (Maphumulo and Bhengu, 2019). However, this situation is not unique to South Africa. In Zimbabwe, provider-level barriers to screening 
perinatal women attending primary healthcare facilities for depression included nurses' lack of time and inadequate training, and lack of privacy making patients less likely to disclose psychological distress (January and Chimbari, 2018). While the lack of privacy was not identified as a barrier in the facilities included in this study, it is a challenge in other PHC facilities in South Africa (Drigo, et al, 2020). Similar findings were reported in a review of studies exploring healthcare workers perceived barriers to screening, referral and management of mental health issues in perinatal women (Bayrampour, et al, 2018), and in reviews of barriers to IPV screening as perceived by healthcare providers (Byatt, et al, 2012; Sprague, et al, 2012; Waalen, et al, 2000). Studies highlighted time constraints, lack of knowledge and training, and insufficient awareness of referral pathways as key provider-level barriers to screening.

Patient-level facilitators included the pregnant women's perceived importance of screening and counselling, while the patient-level barriers included concerns regarding confidentiality when disclosing sensitive information. Service-providers cited cultural beliefs and stigma amongst pregnant women as a barrier. However, none of the pregnant women reported that cultural beliefs influenced their uptake of referrals, even though many alluded to the role of stigma. High levels of mental health stigma have been reported in South Africa. In a qualitative study exploring the experiences of mental health stigma among healthcare providers and users in the North West province of South Africa (Egbe, et al, 2014), the authors reported that users were exposed to stigmatising attitudes of family members, neighbours, friends, church members and the general community. Similarly, a study in Massachusetts USA reported that perinatal women were reluctant to disclose their mental health issues due to stigma and the fear of being judged as an unfit parent, resulting in missed appointments and poor uptake of services (Byatt, et al, 2012).

Our study has strengths and limitations. We have both the perceptions of service providers and service users from the same clinics. However, our service users were interviewed when attending the clinic for their first antenatal appointment, making it difficult to link their perceptions of care with the specific healthcare providers we interviewed. Our study may be limited by social desirability bias, especially regarding the pregnant women's responses to questions on domestic violence, and service providers' responses to their role in providing care.

Several strategies can be used to mitigate the challenges we have identified and strengthen the health system. ANC nurses require training on administering the mental health screening questionnaire to decrease their feelings of discomfort with mental health issues. As nurses' time with patients is limited, the mental health screening questionnaire could be administered in a patient centred manner (Kitson, et al, 2013). ANC nurses could be trained to routinely enquire about their patients' feelings and anxieties while examining them, instead of completing the questionnaire as a ticking exercise while completing the required documentation linked to the consultation. Standardised referral pathways and processes, specific to each facility, need to be developed and disseminated widely to ensure that both healthcare workers and patients are aware of the services available and how to access them. To lessen the burden of specialised mental healthcare providers such as social workers and mental health nurses, a cadre of lay healthcare workers could be identified and trained to provide basic evidence-based problem-solving 
counselling (Boisits, et al, 2021) to women with mild symptoms of depression or anxiety. The limitations of the MCR will need to be addressed at a policy level to encourage healthcare providers to detect symptoms of depression, anxiety and experiences of domestic violence in all pregnant women, at all clinic visits. Intervention studies aimed at strengthening the healthcare system with regards to detection, referral and treatment at MOUs in South Africa are needed.

\section{Conclusion}

Facilitators identified by healthcare workers and pregnant women included the importance of detecting women with symptoms of CMDs and experiences of domestic violence and the availability of a mental health screening questionnaire, yet detection rates at the MOUs were low. Several barriers were identified at the system-level, these related to aspects of the mental health screening questionnaire, and the lack of a screening questionnaire to identify experiences of domestic violence. Provider-level barriers included healthcare workers discomfort in dealing with mental health issues, their limited time available, heavy patient load and lack of awareness of available referral pathways. Patient-level barriers included patients' concerns about the lack of confidentiality, and feelings of shame related to experiences of domestic violence. While the system-level barriers need to be addressed at a policy level, the patient- and providerlevel barriers will be used by the ASSET study to inform the development of a health systems strengthening intervention, to be piloted in MOUs and basic antenatal care clinics in Cape Town.

\section{Declarations}

\section{Author statement}

Authors contributions: All authors provided input in the design of the study. ZA managed the data collection and developed the coding framework. ZA and SB analysed the qualitative interviews. ZA performed the data analysis and prepared the first draft of the manuscript. All authors reviewed the manuscript and providing input. All authors approved the final version of the manuscript.

Funding: This work was supported by the National Institute for Health Research (NIHR) Global Health Research Unit on Health System Strengthening in Sub-Saharan Africa, King's College London [GHRU 16/136/54].

Competing interests: The authors have no competing interests to declare.

Acknowledgements: We are grateful to the healthcare workers and pregnant women who participated in the study for generously giving their time and energy to complete interviews.

\section{References}

Abrahams, Z., Lund, C., Field, S., Honikman, S., 2018. Factors associated with household food insecurity and depression in pregnant South African women from a low socio-economic setting: a cross-sectional 
study. Soc. Psychiatry Psychiatr. Epidemiol., 1-10.

Abrahams, Z., Schneider, M., Field, S., Honikman, S., 2019. Validation of a brief mental health screening tool for pregnant women in a low socio-economic setting. BMC psychology. 7, 77.

Adhikari, M., 2005. Not White enough, Not Black enough: Racial Identity in the South African Coloured Community. Ohio University Press.

ASSET, 2020. Maternal mental health and violence against women in South Africa. 2020.

Baranov, V., Bhalotra, S., Biroli, P., Maselko, J., 2020. Maternal depression, women's empowerment, and parental investment: evidence from a randomized controlled trial. Am. Econ. Rev. 110, 824-859.

Bayrampour, H., Hapsari, A.P., Pavlovic, J., 2018. Barriers to addressing perinatal mental health issues in midwifery settings. Midwifery. 59, 47-58.

Bazeley, P. and Jackson, K., 2013. Qualitative data analysis with NVivo.

Boisits, S., Abrahams, Z., Schneider, M., Honikman, S., Kaminer, D., Lund, C., 2021. Developing a tasksharing psychological intervention to treat mild to moderate symptoms of perinatal depression and anxiety in South Africa: a mixed-method formative study. International Journal of Mental Health Systems. $15,1-14$.

Byatt, N., Biebel, K., Lundquist, R.S., Moore Simas, T.A., Debordes-Jackson, G., Allison, J., Ziedonis, D., 2012. Patient, provider, and system-level barriers and facilitators to addressing perinatal depression. Journal of Reproductive and Infant Psychology. 30, 436-449.

Chen, P., Rovi, R., Pan, K., Johnson, M., 2013. Costs Effectiveness of Domestic Violence Screening in Primary Care Settings: A Comparison of 3 Methods. J Community Med Health Educ. 3, 21610711.1000253.

Coovadia, H., Jewkes, R., Barron, P., Sanders, D., Mclntyre, D., 2009. The health and health system of South Africa: historical roots of current public health challenges. The Lancet. 374, 817-834.

Cox, J.L., Holden, J.M., Sagovsky, R., 1987. Detection of postnatal depression: development of the 10-item Edinburgh Postnatal Depression Scale. Br J Psychiatry. 150, 782-786.

Davis, E.C., Rotheram-Borus, M.J., Weichle, T.W., Rezai, R., Tomlinson, M., 2017. Patterns of alcohol abuse, depression, and intimate partner violence among township mothers in South Africa over 5 years. AIDS and Behavior. 21, 174-182.

De Bruin, G.P., Swartz, L., Tomlinson, M., Cooper, P.J., Molteno, C., 2004. The factor structure of the Edinburgh Postnatal Depression scale in a South African peri-urban settlement. S. Afr. J. Psychol. 34, 113-121. 
Dennis, C., Falah-Hassani, K., Shiri, R., 2017. Prevalence of antenatal and postnatal anxiety: systematic review and meta-analysis. The British Journal of Psychiatry. 210, 315-323.

Department of Health, 2013. National Mental Health Policy Framework and Strategic Plan, 2013-2020 .

Department of Health, 2018. Maternity Case Record. 2020.

Docrat, S., Besada, D., Cleary, S., Daviaud, E., Lund, C., 2019. Mental health system costs, resources and constraints in South Africa: a national survey. Health Policy Plan. 34, 706-719.

Drigo, L., Luvhengo, M., Lebese, R.T., Makhado, L., 2020. Attitudes of Pregnant Women Towards Antenatal Care Services Provided in Primary Health Care Facilities of Mbombela Municipality, Mpumalanga Province, South Africa. The Open Public Health Journal. 13.

Egbe, C.O., Brooke-Sumner, C., Kathree, T., Selohilwe, O., Thornicroft, G., Petersen, I., 2014. Psychiatric stigma and discrimination in South Africa: perspectives from key stakeholders. BMC Psychiatry. 14, 1-14.

English, R., Peer, N., Honikman, S., Tugendhaft, A., Hofman, K.J., 2017. 'First 1000 days' health interventions in low-and middle-income countries: alignment of South African policies with high-quality evidence. GLOBAL HEALTH. 10, 1340396.

Field, S., Onah, M., van Heyningen, T., Honikman, S., 2018. Domestic and intimate partner violence among pregnant women in a low resource setting in South Africa: a facility-based, mixed methods study. BMC Womens Health. 18, 1-13.

Gale, N.K., Heath, G., Cameron, E., Rashid, S., Redwood, S., 2013. Using the framework method for the analysis of qualitative data in multi-disciplinary health research. BMC medical research methodology. 13, $1-8$.

Garman, E.C., Schneider, M., Lund, C., 2019. Perinatal depressive symptoms among low-income South African women at risk of depression: trajectories and predictors. BMC pregnancy and childbirth. 19, 202.

Gelaye, B., Rondon, M.B., Araya, R., Williams, M.A., 2016. Epidemiology of maternal depression, risk factors, and child outcomes in low-income and middle-income countries. The Lancet Psychiatry. 3, 973982.

Gwet, K.L., 2014. Handbook of Inter-Rater Reliability: The Definitive Guide to Measuring the Extent of Agreement among Raters. Advanced Analytics, LLC.

Hartley, M., Tomlinson, M., Greco, E., Comulada, W.S., Stewart, J., Le Roux, I., Mbewu, N., Rotheram-Borus, M.J., 2011. Depressed mood in pregnancy: prevalence and correlates in two Cape Town peri-urban settlements. Reproductive health. 8, 9. 
Honikman, S., Van Heyningen, T., Field, S., Baron, E., Tomlinson, M., 2012. Stepped care for maternal mental health: a case study of the perinatal mental health project in South Africa. PLoS Med. 9, e1001222.

January, J. and Chimbari, M.J., 2018. Opportunities and obstacles to screening for perinatal depression among women in Zimbabwe: A narrative review of literature. South African Journal of Psychiatry. 24.

Jarde, A., Morais, M., Kingston, D., Giallo, R., MacQueen, G.M., Giglia, L., Beyene, J., Wang, Y., McDonald, S.D., 2016. Neonatal outcomes in women with untreated antenatal depression compared with women without depression: a systematic review and meta-analysis. JAMA psychiatry. 73, 826-837.

Kitson, A., Marshall, A., Bassett, K., Zeitz, K., 2013. What are the core elements of patient-centred care? A narrative review and synthesis of the literature from health policy, medicine and nursing. J. Adv. Nurs. 69, 4-15.

Klein, D., 2018. Implementing a general framework for assessing interrater agreement in Stata. The Stata Journal. 18, 871-901.

Lawrie, T., Hofmeyr, G., De Jager, M., Berk, M., 1998. Validation of the Edinburgh Postnatal Depression Scale on a cohort of South African women. S Afr Med J. 88, 1340-1344.

Malan, M., Spedding, M.F., Sorsdahl, K., 2018. The prevalence and predictors of intimate partner violence among pregnant women attending a midwife and obstetrics unit in the Western Cape. Global Mental Health. 5.

Manikkam, L. and Burns, J.K., 2012. Antenatal depression and its risk factors: an urban prevalence study in KwaZulu-Natal. South African Medical Journal. 102, 940-944.

Maphumulo, W.T. and Bhengu, B.R., 2019. Challenges of quality improvement in the healthcare of South Africa post-apartheid: A critical review. Curationis. 42, 1-9.

Maringira, G. and Masiya, T., 2018. Persistence of Youth Gang Violence in South Africa. The African Review. 45, 164-179.

Pluddemann, A., 2012. The SACENDU project: monitoring alcohol and drug abuse trends in South Africa. International Journal of Psychology. 47.

Rahman, A., Bunn, J., Lovel, H., Creed, F., 2007. Association between antenatal depression and low birthweight in a developing country. Acta Psychiatr. Scand. 115, 481-486.

Rahman, A., Fisher, J., Bower, P., Luchters, S., Tran, T., Yasamy, M.T., Saxena, S., Waheed, W., 2013. Interventions for common perinatal mental disorders in women in low-and middle-income countries: a systematic review and meta-analysis. Bull. World Health Organ. 91, 593-601I. 
Redinger, S., Norris, S., Pearson, R., Richter, L., Rochat, T., 2018. First trimester antenatal depression and anxiety: prevalence and associated factors in an urban population in Soweto, South Africa. Journal of developmental origins of health and disease. 9, 30-40.

Schneider, M., Baron, E., Davies, T., Munodawafa, M., Lund, C., 2018. Patterns of intimate partner violence among perinatal women with depression symptoms in Khayelitsha, South Africa: A longitudinal analysis. Global Mental Health. 5.

Sivarajasingam, V., 2021. Routine screening for domestic abuse. British Journal of General Practice. 71, 173-173.

Skapinakis, P., Kroenke, K., Spitzer, R., Williams, J., 2007. The 2-item Generalized Anxiety Disorder scale had high sensitivity and specificity for detecting GAD in primary care. Evid Bas Med. 12, 149.

South African History Online, 2020. Cape Flats, Cape Town. 2020.

Sprague, S., Madden, K., Simunovic, N., Godin, K., Pham, N.K., Bhandari, M., Goslings, J., 2012. Barriers to screening for intimate partner violence. Women Health. 52, 587-605.

Statistics South Africa, 2017. Poverty trends in South Africa: An examination of absolute poverty between 2006 and 2015.

Tomlinson, M., O’Connor, M.J., Le Roux, I.M., Stewart, J., Mbewu, N., Harwood, J., Rotheram-Borus, M.J., 2014. Multiple risk factors during pregnancy in South Africa: the need for a horizontal approach to perinatal care. Prevention Science. 15, 277-282.

van Heyningen, T., Myer, L., Onah, M., Tomlinson, M., Field, S., Honikman, S., 2016. Antenatal depression and adversity in urban South Africa. J. Affect. Disord. 203, 121-129.

van Heyningen, T., Honikman, S., Myer, L., Onah, M.N., Field, S., Tomlinson, M., 2017. Prevalence and predictors of anxiety disorders amongst low-income pregnant women in urban South Africa: a crosssectional study. Archives of women's mental health. 20, 765-775.

Waalen, J., Goodwin, M.M., Spitz, A.M., Petersen, R., Saltzman, L.E., 2000. Screening for intimate partner violence by health care providers: barriers and interventions. Am. J. Prev. Med. 19, 230-237.

Whooley, M., 2016. Whooley questions for depression screening. 2018.

Woody, C., Ferrari, A., Siskind, D., Whiteford, H., Harris, M., 2017. A systematic review and meta-regression of the prevalence and incidence of perinatal depression. J. Affect. Disord. 219, 86-92.

World Health Organisation, 2012. Zero Draft Global Mental Health Action Plan 2013 - 2020. 